\title{
THz Quantum Cascade Laser Operating Frequency Comb Over Near-full-current Dynamic Range
}

\section{Yu Ma}

Institute of Semiconductors Chinese Academy of Sciences

Yuan-Yuan Li

Institute of Semiconductors Chinese Academy of Sciences

Junqi Liu ( $\sim$ jqliu@semi.ac.cn )

Institute of Semiconductors Chinese Academy of Sciences

https://orcid.org/0000-0003-1654-6174

\section{Ning Zhuo}

Institute of Semiconductors Chinese Academy of Sciences

\section{Wei-Jiang Li}

Institute of Semiconductors Chinese Academy of Sciences

\section{Ke Yang}

Institute of Semiconductors Chinese Academy of Sciences

Jin-Chuan Zhang

Institute of Semiconductors Chinese Academy of Sciences

\section{Shen-Qiang Zhai}

Institute of Semiconductors Chinese Academy of Sciences

\section{Li-Jun Wang}

Institute of Semiconductors Chinese Academy of Sciences

Shu-Man Liu

Institute of Semiconductors Chinese Academy of Sciences

\section{Feng-Qi Liu}

Institute of Semiconductors Chinese Academy of Sciences

\section{Research Article}

Keywords: terahertz, quantum cascade laser, optical frequency combs

Posted Date: September 22nd, 2021

DOI: https://doi.org/10.21203/rs.3.rs-907795/v1

License: (c) (i) This work is licensed under a Creative Commons Attribution 4.0 International License. Read Full License 



\section{$\mathrm{THz}$ quantum cascade laser operating frequency comb over near-full-current dynamic range}

Yu Ma ${ }^{1,2,}$, Yuan-Yuan Li ${ }^{1,3, *}$, Jun-Qi Liu ${ }^{1,2,4}$, Ning Zhuo ${ }^{1,5}$, Wei-Jiang Li ${ }^{1,2}$, Ke Yang ${ }^{1,2}$, Jin-Chuan Zhang ${ }^{1}$,Shen-Qiang

Zhai $^{1}$,Li-Jun Wang ${ }^{1,2}$, Shu-Man Liu ${ }^{1,2}$, and Feng-Qi Liu ${ }^{1,2,3}$

Electronic emails:

Yu Ma: mayu@semi.ac.cn

Yuan-Yuan Li: liyuanyuan@semi.ac.cn

Jun-Qi Liu:jqliu@semi.ac.cn(corresponding author)

Ning Zhuo: zhuoning@semi.ac.cn(corresponding author)

Wei-Jiang Li: liweijiang@semi.ac.cn

Ke Yang: yangke17@semi.ac.cn

Jin-Chuan Zhang: zhangjinchuan@semi.ac.cn

Shen-Qiang Zhai: zsqlzsmbj@semi.ac.cn

Li-Jun Wang: ljwang@semi.ac.cn

Shu-Man Liu: $\underline{\text { liusm@semi.ac.cn }}$

Feng-Qi Liu: fqliu@semi.ac.cn

${ }^{1}$ Key Laboratory of Semiconductor Materials Science, Institute of Semiconductors, Chinese Academy of Sciences, Beijing Key Laboratory of Low Dimensional Semiconductor Materials and Devices, P.O. Box 912, Beijing100083, China

${ }^{2}$ Center of Materials Science and Optoelectronics Engineering, University of Chinese Academy of Sciences, Beijing 100049, 
China

${ }^{3}$ Division of Quantum Materials and Devices, Beijing Academy of Quantum Information Sciences, Beijing 100193, China

4jqliu@semi.ac.cn

${ }^{5}$ zhuoning@semi.ac.cn

*Yu Ma and Yuan-Yuan Li contributed equally to this work.

\section{Abstract}

We report a terahertz quantum cascade laser frequency comb (THz QCL FC) with low threshold current density, high power, and wide current dynamic range. The active region design with the semi-insulated surface plasma waveguide is beneficial to optimize the gain dispersion value and temperature stability. At $10 \mathrm{~K}$, the comb with 3 $\mathrm{mm}$-long and $150-\mu \mathrm{m}$-wide is capable of emitting $22 \mathrm{~mW}$ with the threshold current density $\mathrm{J}_{\text {th }}=64.4 \mathrm{~A} \cdot \mathrm{cm}^{-2}$. The total spectral emission is of about $300 \mathrm{GHz}$ centered around $4.6 \mathrm{THz}$. Without any extra dispersion compensation measures, the intermode beatnote map reveals stable frequency comb operating within a current dynamic range more than $97 \%$ and the narrowest beatnote linewidth is $7.2 \mathrm{kHz}$. The stable FC operation of a freerunning THz QCL makes our device an ideal source for further development of dual-comb spectroscopy.

Keywords: terahertz; quantum cascade laser; optical frequency combs;

\section{Introduction}

Optical frequency combs (OFCs) composed of a series of equidistant spectral lines in the frequency domain[1] have many revolutionary applications due to their high stability and low phase noise, such as in high resolution and precision spectral measurement, high capacity laser communication and other related fields[2]. In the terahertz (THz) range, most of the molecular rotation and vibration frequencies are concentrated, so the OFCs have attracted much attention for a considerable prospect in sensing, fast and high-resolution spectroscopy and metrology, 
nondestructive biological tissue testing, gas detection and so on[3,4]. The development of THz OFCs is, nevertheless, once greatly limited by the lack of high-power and compact light source. In recent years, the continuous maturity of quantum cascade lasers (QCLs) [5-8] from mid-infrared to the THz spectral range provides an opportunity for advancing the development of OFCs by a mechanism of intracavity nonlinear four-wave mixing and injection locking[9]. However, emergence of more promising THz QCL OFCs toward yielding more stable comb operating, wider current dynamic range and spectral range[10-12], higher output power[13] and narrower beam divergence is still expected for further application.

Due to the stable waveguide loss, QCL with the double metal (MM) waveguide structure is generally used to form THz OFCs. A dispersion compensator $[14,15]$ was integrated into the waveguide to minimize the dispersion to form a stable optical comb in the large current range. Heterogeneous multistacked active regions [16,17] technology was used to broaden the gain to obtain an octave-spanning emission. But, the optical power of $\mathrm{THz}$ OFCs based on MM waveguide is usually limited to only a few mWs [18], threshold current density exceeds 100 $\mathrm{A} \cdot \mathrm{cm}^{-2}$, and, what is more, the longitudinal far field divergence angle is approximately $180^{\circ}[19]$. On the contrary, THz QCLs with the semi-insulated surface plasma (SISP) waveguide [5,20,21]show advantages in these aspects. However, its waveguide loss causes a larger group velocity dispersion (GVD), so the stable optical comb operation is generally formed only in some intermittent current range. On the other hand, limited by the device structure, it is difficult to integrate efficient dispersion compensator on SISP waveguide.

Here, in this paper, we present a versatile band design way to control the gain dispersion and temperature stability, permitting comb formation over near-full-current dynamic range of the laser. This work finally prepares a low threshold current density and high-power THz QCL OFCs capable of emitting $22 \mathrm{~mW}$ at $4.6 \mathrm{THz}$ with the threshold current density $64.4 \mathrm{~A} \cdot \mathrm{cm}^{-2}$. Remarkably, the intermode beatnote map reveals free-running of the $\mathrm{THz}$ QCL operating OFC over the near-full-current range (>97\%). 


\section{Design and simulation}

The material structure of our comb is grown by molecular beam epitaxy (MBE) on a semi-insulating (SI) GaAs substrate. The active region design of the homogeneous gain medium with 180 periods is a slightly modified version of the hybrid bound-to-continuum transition and resonant phonon extraction as described in Ref. [20].

The layer sequence of one period starting from the injection barrier (in $\mathrm{nm}$ ) is 5.3/9.8/1.1/11/3.5/9.2/4.8/17.3, where the barriers in bold are $\mathrm{Al}_{0.22} \mathrm{Ga}_{0.78} \mathrm{As}$ and the wells are GaAs. The underlined GaAs well is Si-doped to $1 \times 10^{16} \mathrm{~cm}^{-3}$. A conduction band diagram of the active region is shown in Fig. 1. Different from the literature mentioned above, the performance of the epitaxial wafers is improved by optimizing the structure of active region, including the material thickness, composition and doping concentration. To suppress the carrier leakage over the AlGaAs barriers, higher barriers with an $\mathrm{Al}$ composition are increased from $15.5 \%$ to $22 \%$. In the experiment, we find that thinner barrier leads to excessive current and serious heating of the device. Properly increasing the barrier thickness reduces the parasitic coupling and the current density, even if the population inversion and gain are lowered. As for the doping concentration, excessively high doping concentration causes large current and serious heating. To ensure the gain, the doping concentration is regulated. In this scheme, we also have to consider the intermodule current leakage tunnelling. For one period, electrons are injected into the upper laser level 5 from the injector level g' by resonant tunnelling. And then the lasers are generated through a diagonal transition. The lower lasing level 4 and other levels (3 and 2) forming the miniband are depopulated through LO-phonon scattering into a ground state $\mathrm{g}$, which is the origin of a broad spectrum and gives a flatter gain to reduce or compensate the dispersion. The structure is designed to be a clean five-level system, making most of the electrons in the $n \_$th module remain within those five levels $(5,4,3,2, \mathrm{~g})$. When the bias is set to align the energy levels g' and 5 (injection alignment) in traditional scheme, a noticeable spatial overlap exists between those two levels and the higher-energy bound states denoted by the energy level 6 and 7, as shown in Fig.1. We aim to minimize the 
electron-LO-phonon absorption into the doublet levels (6 and 7) from the g'-5 levels. Using a wide well pushes down the energy level 6 and the high $\mathrm{Al}$ component pushes up the energy level 7 to stay away from the energy levels g' and 5, which reduce the effect of leakage channels. According to the above adjustment, the structure with low current density is obtained.

By modifying the band structure, a 4.6 THz QCL structure with a wide emitting range is obtained. But the THz wave with different frequencies propagating at different group velocities in the same medium causes the group velocity dispersion, which directly affects the stability and phase noise of the combs. To ensure the THz QCL operate in the OFC mode, GVD is numerically simulated, including the contributions from the material, the waveguide and the gain. For the material originated GVD of the AlGaAs/GaAs heterostructure QCL, we use bulk GaAs to replace it. Since the reststrahlen band have an effect on the QCL emitting range, the index of refraction changes slightly. The relationship can be described as,

$$
\begin{gathered}
\varepsilon(\omega)=\varepsilon_{\infty}+\varepsilon_{\infty} \frac{\omega_{L O}^{2}-\omega_{T O}^{2}}{\omega_{T O}^{2}-\omega\left(\omega+j \gamma_{p h}\right)}, \\
n=\sqrt{\frac{|\varepsilon(\omega)|+\operatorname{Re}(\varepsilon(\omega))}{2}},
\end{gathered}
$$

where, $\varepsilon_{\infty}$ is the high-frequency dielectric constant, $\omega=2 \pi c / \lambda$ is the photon angular frequency, $\gamma_{p h}=$ $240 \mathrm{~m}^{-1}$ is phonon damping constant, and $\omega_{L O}=29210 \mathrm{~m}^{-1}$ and $\omega_{T O}=26870 \mathrm{~m}^{-1}$ are the longitudinal and transverse optical phonon frequencies, respectively. The relationship between the real part of the refractive index and the frequency is solved. For the waveguide originated GVD, the finite element method is used to study the waveguide cross section perpendicular to the direction of the guided mode propagation. According to the DrudeLorentz model, the interactions between the light field and the free carriers, and the light field and the phonons are considered comprehensively[22],

$$
\varepsilon(\omega)=\varepsilon_{\infty}\left(1+\varepsilon_{\text {phonon }}+\varepsilon_{\text {plsma }}\right)=\varepsilon_{\infty}\left(1+\frac{\omega_{L O}^{2}-\omega_{T O}^{2}}{\omega_{T O}^{2}-\omega\left(\omega+j \gamma_{p h}\right)}-\frac{\omega_{p}^{2}}{\omega\left(\omega+j \gamma_{p l}\right)}\right),
$$

where, $\omega_{p}=\sqrt{\frac{N e^{2}}{m^{*} \varepsilon_{\infty} \varepsilon_{0}}}$ is the plasma frequency and $\gamma_{p l}=\frac{e}{m^{*} \mu}$ is the plasmon damping frequency. In this way, 
the refractive index of each material as a function of frequency is determined. Then the waveguide loss of the lasers is calculated with the COMSOL Software as shown in Fig. 2(a). For the gain GVD, it is the most important part of the total GVD. Here, due to the gain clamping effect, when it is greater than the threshold gain, the gain of the device will be clamped to the threshold gain[23,24]. We use the clamped gain to fit the actual working situation to calculate the gain GVD. The gain of the QCL can be calculated by the Fermi's golden rule,

$$
g(\omega)=\frac{e^{2} \Delta n \cdot z^{2} \omega}{\varepsilon_{0} \cdot n_{r e f} \cdot L \cdot c} \frac{\gamma}{(E-\hbar \omega)^{2}+\gamma^{2}}
$$

where, $\Delta n$ is population inversion, $z$ is the dipole matrix element of the optical transition, $n_{\text {ref }}$ is the effective refractive index of the active region, $L$ is the period length of the QCL active region, $E$ is the energy of the optical transition, and $\gamma$ is the level broadening. Near the threshold, the gain equals to the losses. So we consider the influence of the losses on the gain, including the loss of the intersubband absorption, and the waveguide loss and the mirror loss. Then, the threshold gain can be calculated as,

$$
g_{t h}=\alpha_{i}+\left(\alpha_{w}+\alpha_{m}\right) / \Gamma
$$

where, $\Gamma$ is the mode confinement factor, $\alpha_{i}, \alpha_{w}$ and $\alpha_{m}$ are the intersubband absorption, the waveguide loss and mirror loss, respectively. Fig. 2(b) shows the calculated losses and gain. Finally, we evaluate the refractive index deviation as a consequence of the gain and the losses by applying the Kramers-Kronig equation. Now we can figure out the relationship between the GVD and the refractive index,

$$
G V D=\frac{\partial}{\partial \omega} \frac{n(\omega)+\omega \frac{\partial n(\omega)}{\partial \omega}}{c}
$$

where, $n(\omega)$ is the refractive index. Fig. 2(c) shows the calculated GVD, which indicates a small GVD value in the spectral coverage. In a wide emitting range, dispersion can cause the spacing of adjacent longitudinal modes to change with different frequencies, so that the frequency comb device cannot be guaranteed to present a single beatnote signal, which would result in the device operating in non-OFC mode. When the dispersion is unmanageable, the farther from the center frequency the mode is, the greater the frequency offset will be. In Fig. 
2(c), the small simulated GVD value ensures that the device has the potential to be a proper OFC.

To verify the simulation results, the device based on the SISP waveguide was fabricated[21]. The photolithography and wet etching were used to obtain the ridges. Two $20-\mu \mathrm{m}$-wide stripes in the ridges and the area of the bottom contact layer were covered with $\mathrm{Ge} / \mathrm{Au} / \mathrm{Ni} / \mathrm{Au}$ under thermal annealing at $360^{\circ} \mathrm{C}$ for $15 \mathrm{~s}$. Then, 5-nm-thick Ti and 200-nm-thick Au were deposited covering both the top of the ridge and the bottom contact layer to allow wire bonding. At last, the substrate was thinned down to $\sim 150 \mu \mathrm{m}$ and a device with a 3-mm-long cavity and $150-\mu \mathrm{m}$-wide ridge was fabricated. The positive electrode was connected to the SMA center pin and the negative electrode was grounded. Finally, the device with lens collimation is packaged into the test system for subsequent tests, as shown in Fig. 3.

\section{Results and discussion}

The light-current-voltage (L-I-V) characteristic curve as shown in Fig. 4 are measured while driving the QCL in continuous-wave (CW) by a power supply (THORLABS ITC4005 QCL) as a function of the setpoint temperature $\left(T_{s}\right)$, which show the laser action up to a maximum setpoint temperature of $T_{s}=65 \mathrm{~K}$. At $T_{s}=10 \mathrm{~K}$, the $\mathrm{CW}$ threshold current density $\mathrm{Jth}=64.4 \mathrm{~A} \cdot \mathrm{cm}^{-2}$, the maximum current density $\mathrm{Jmax}=142.2 \mathrm{~A} \cdot \mathrm{cm}^{-2}$, the operational dynamic range $\mathrm{J}_{\mathrm{dr}}=\mathrm{J}_{\max } / \mathrm{J}_{\mathrm{th}}=2.2$, and the maximum peak output power is $22 \mathrm{~mW}$. The spectra of devices were tested by a Fourier transform infrared spectrometer with a resolution of $0.2 \mathrm{~cm}^{-1}$. The L-I-V characteristics of the devices were tested by a calibrated thermopile detector. The laser was mounted on a holder containing a thermistor combined with a thermoelectric cooler to monitor and adjust the sub-mount temperature. The emitted optical power was measured with the calibrated thermopile detector placed in front of the laser facet without any correction.

The CW Fourier transform infrared spectra (FTIR) acquired under vacuum with a $0.2 \mathrm{~cm}^{-1}$ spectral resolution 
while progressively increasing the current indicates that the device is initially emitting a single frequency mode $(\sim 4.4 \mathrm{THz})$ at the threshold and then turns to multimode with a series of equidistant optical modes (Fig. 5), spaced by the cavity round-trip frequency. The overall spectral coverage reaches $300 \mathrm{GHz}(4.4-4.7 \mathrm{THz})$. The central frequency at $\sim 4.6 \mathrm{THz}$ matches the design of the energy band.

In order to explore the comb operation, an intermode beatnote emitting from the device is detected to characterize the coherence properties by an RF spectral analyzer (MXA Signal Analyzer N9020A). In Fig. 6(a), without any dispersion compensation, a single beatnote signal is presented from $12.2 \mathrm{GHz}$ to $11.9 \mathrm{GHz}$. This confirms that the comb operation is achieved with the current range of more than $97 \%$ (from $0.3 \mathrm{~A}$ to $0.7 \mathrm{~A}$ ), or the near-full-current range. The intensity of the beatnote signal increases with the increase of current. The highest signal noise ratio is $35 \mathrm{dBm}$, as shown in Fig. $6(\mathrm{~b})$; and the narrowest beatnote linewidth at $0.6 \mathrm{~A}$ is $7.2 \mathrm{KHz}$, as shown in Fig. 6(c). The resolution bandwidth is set as 100KHz. The qualified coherence properties of the combs are suitable for the simulation calculation, especially flattening dispersion control.

We also investigate the effect of low threshold current density on OFC. For the OFC devices, only a small part of the excitation energy provided by the power source is converted into the light energy, with most into the heat energy. A device with a low threshold current density can reduce the total heat consumption, minimize the negative impact of temperature rise on the gain and achieve better OFC operation in a more stable environment. For this purpose, we calculated the effect of temperature on the gain and the results are shown in Fig. 7. In the emitting range, the temperature fluctuation is fairly small, only from $6 \mathrm{~K}$ to $10 \mathrm{~K}$. Considering that the heat sink temperature is detected, the core region temperature of the device will be at least $20 \mathrm{~K}$ higher, so the temperature of $25-65 \mathrm{~K}$ is taken for the simulation, and it is also found that the temperature fluctuation has caused only $0.6 \%$ on the gain at 26-30K. It can be seen from Fig. 7 that the calculated gain decreases with the increase of temperature, which is mainly due to the decrease of the population inversion with the increase of temperature. The rate of the 
gain decrease is estimated to be $0.31 \mathrm{~cm}^{-1} \cdot \mathrm{K}^{-1}$ at $25-65 \mathrm{~K}$, which shows great temperature stability.

\section{Conclusions}

We have developed a low dispersion THz QCL for optical frequency comb (OFC) purpose with the semiinsulating surface-plasmon (SISP) waveguide structure by adjusting the material thickness, composition and doping concentration of the active region design of the homogeneous gain medium based on the hybrid bound-tocontinuum transition and resonant phonon extraction. For the device fabricated with 3 -mm-long cavity and $150-$ $\mu \mathrm{m}$-wide ridge, the maximum power achieves $22 \mathrm{~mW}$ emitting at $\sim 4.6 \mathrm{THz}$ with $\mathrm{J}_{\mathrm{th}}=64.4 \mathrm{~A} \cdot \mathrm{cm}^{-2}$ and a total spectral emission of about $300 \mathrm{GHz}$ at $10 \mathrm{~K}$. Without any dispersion compensation, the comb operation is achieved over the near-full-current (>97\%) dynamic range and the narrowest beat line width is $7.2 \mathrm{kHz}$. The simulation results show that the device has low dispersion value and high temperature stability, ensuring the proper OFC operation.

\section{Abbreviations}

OFCs: optical frequency combs; THz: terahertz; QCL: quantum cascade laser; MM: double metal; SISP: semiinsulated surface plasma; MBE: molecular beam epitaxy; SI: semi-insulating; GVD: group velocity dispersion; LIV: light-current-voltage; CW: continuous wave; $\mathrm{T}_{\mathrm{s}}$ : setpoint temperature; FTIR: Fourier transform infrared spectra;

\section{Availability of data and materials}

All data are fully available without restriction. 


\section{Competing interests}

The authors declare that they have no competing interests.

\section{Funding}

This work was supported by the National Natural Science Foundation of China under Grant Nos. 61734006, 61835011, 61991430, and the Key Program of the Chinese Academy of Sciences under Grant Nos. XDB43000000, YJKYYQ20190002, QYZDJ-SSW-JSC027.

\section{Authors' contributions}

Yu Ma and Yuan-Yuan Li contributed equally to this work. YM designed the devices structure, calculated the theoretical model, and wrote the paper. YYL fabricated the devices and performed the testing, JQL and NZ provided the concept, polished the paper, and supervised the project. KY and WJL assisted with the theoretical model and testing. SML, SQZ and JCZ improved the design. NZ and LJW modulated the active region structure and completed the MBE growth. FQL supervised the project. All authors read and approved the final manuscript.

\section{Acknowledgements}

The authors would like to thank Ping Liang and Ying Hu for their help in device processing.

\section{References}

1. J. Hillbrand, A. M. Andrews, H. Detz, G. Strasser, and B. Schwarz, "Coherent injection locking of quantum cascade laser frequency combs," NAT PHOTONICS 13, 101-104 (2019).

2. L. Consolino, M. Nafa, M. De Regis, F. Cappelli, K. Garrasi, F. P. Mezzapesa, L. Li, A. G. Davies, E. H. Linfield, M. S. Vitiello, S. Bartalini, and P. De Natale, "Quantum cascade laser based hybrid dual comb spectrometer," Communications Physics 3 (2020).

3. L. A. Sterczewski, J. Westberg, Y. Yang, D. Burghoff, J. Reno, Q. Hu, and G. Wysocki, "Terahertz hyperspectral imaging with dual chip-scale combs," OPTICA 6, 766-771 (2019). 
4. K. Garrasi, F. P. Mezzapesa, L. Salemi, L. Li, L. Consolino, S. Bartalini, P. De Natale, A. G. Davies, E. H. Linfield, and M. S. Vitiello, "High Dynamic Range, Heterogeneous, Terahertz Quantum Cascade Lasers Featuring Thermally Tunable Frequency Comb Operation over a Broad Current Range," ACS PHOTONICS 6, 73-78 (2019).

5. J. Liu, J. Chen, F. Liu, L. Li, L. Wang, and Z. Wang, "Terahertz Quantum Cascade Laser Operating at 2.94 THz," CHINESE PHYS LETT 27 (2010).

6. S. Kumar, Q. Hu, and J. L. Reno, "186 K operation of terahertz quantum-cascade lasers based on a diagonal design," APPL PHYS LETT 94, 131105 (2009).

7. T. Wang, J. Liu, J. Chen, Y. Liu, F. Liu, L. Wang, and Z. Wang, "Continuous-Wave Operation of Terahertz Quantum Cascade Lasers at 3.2 THz," CHINESE PHYS LETT 30 (2013).

8. Y. Li, J. Liu, F. Liu, J. Zhang, S. Zhai, N. Zhuo, L. Wang, S. Liu, and Z. Wang, "High power-efficiency terahertz quantum cascade laser," CHINESE PHYS B 25 (2016).

9. A. Hugi, G. Villares, S. Blaser, H. C. Liu, and J. Faist, "Mid-infrared frequency comb based on a quantum cascade laser," NATURE 492, 229-233 (2012).

10. P. Tzenov, D. Burghoff, Q. Hu, and C. Jirauschek, "Analysis of Operating Regimes of Terahertz Quantum Cascade Laser Frequency Combs," IEEE T THZ SCI TECHN 7, 351-359 (2017).

11. F. P. Mezzapesa, V. Pistore, K. Garrasi, L. Li, A. G. Davies, E. H. Linfield, S. Dhillon, and M. S. Vitiello, "Tunable and compact dispersion compensation of broadband THz quantum cascade laser frequency combs," OPT EXPRESS 27, 20231 (2019).

12. A. Forrer, L. Bosco, M. Beck, J. Faist, and G. Scalari, "RF Injection of THz QCL Combs at 80 K Emitting over 700 GHz Spectral Bandwidth," Photonics 7, 9 (2020).

13. Z. Li, W. Wan, K. Zhou, X. Liao, S. Yang, Z. Fu, J. C. Cao, and H. Li, "On-Chip Dual-Comb Source Based on Terahertz Quantum Cascade Lasers Under Microwave Double Injection," PHYS REV APPL 12 (2019).

14. D. Burghoff, T.-Y. Kao, N. Han, C. W. I. Chan, X. Cai, Y. Yang, D. J. Hayton, J.-R. Gao, J. L. Reno, and Q. Hu, “Terahertz laser frequency combs,” Nature Photonics 8, 462-467 (2014).

15. F. Wang, H. Nong, T. Fobbe, V. Pistore, S. Houver, S. Markmann, N. Jukam, M. Amanti, C. Sirtori, S. Moumdji, R. Colombelli, L. Li, E. Linfield, G. Davies, J. Mangeney, J. Tignon, and S. Dhillon, "Short Terahertz Pulse Generation from a Dispersion Compensated Modelocked Semiconductor Laser," LASER PHOTONICS REV 11, 1700013 (2017).

16. D. Bachmann, M. Rösch, C. Deutsch, M. Krall, G. Scalari, M. Beck, J. Faist, K. Unterrainer, and J. Darmo, "Spectral gain profile of a multi-stack terahertz quantum cascade laser," APPL PHYS LETT 105, 181118 (2014).

17. L. H. Li, K. Garrasi, I. Kundu, Y. J. Han, M. Salih, M. S. Vitiello, A. G. Davies, and E. H. Linfield, "Broadband heterogeneous terahertz frequency quantum cascade laser," ELECTRON LETT 54, 1229-1230 (2018).

18. D. Bachmann, M. Rösch, M. J. Süess, M. Beck, K. Unterrainer, J. Darmo, J. Faist, and G. Scalari, "Short pulse generation and mode control of broadband terahertz quantum cascade lasers," OPTICA 3, 1087 (2016).

19. Y. Zhu, H. Li, W. Wan, T. Zhou, and J. Cao, "Far-field analysis of third-order distributed feedback terahertz quantum cascade lasers," ACTA PHYS SIN-CH ED 66 (2017).

20. Y. Li, J. Liu, T. Wang, F. Liu, S. Zhai, J. Zhang, N. Zhuo, L. Wang, S. Liu, and Z. Wang, "High-Power and High-Efficiency Operation of Terahertz Quantum Cascade Lasers at 3.3 THz," CHINESE PHYS LETT 32 (2015).

21. K. Zhou, H. Li, W. J. Wan, Z. P. Li, X. Y. Liao, and J. C. Cao, "Ridge width effect on comb operation in terahertz quantum cascade lasers," APPL PHYS LETT 114, 191106 (2019). 
22. G. Rehouma, C. A. Evans, Z. Ikonić, D. Indjin, and P. Harrison, "Comparison of SiO2, Si3N4, As2S3, and $\mathrm{Ge} 0.25 \mathrm{Se} 0.75$ dielectric layers for InP- and GaAs-based material systems for midinfrared quantum cascade laser waveguides," J APPL PHYS 106, 53104 (2009).

23. H. Li, J. C. Cao, and J. T. Lue, "Monte Carlo simulation of carrier transport and output characteristics of terahertz quantum cascade lasers," J APPL PHYS 103 (2008).

24. K. Zhou, H. Li, W. Wan, Z. Li, and J. Cao, "Group velocity dispersion analysis of terahertz quantum cascade laser frequency comb," ACTA PHYS SIN-CH ED 68 (2019).

\section{Captions}

Fig. 1. Conduction band diagram and energy level profile of a 4.6 THz QCL structure. An electric field of 9.3 $\mathrm{kV} / \mathrm{cm}$ is applied to the structure. The red arrow marks the radiative transition. The upper laser level (level 5) and the lower lasing level (level 4) are shown in red and green, respectively. The ground state of the injector (level g) is shown in blue. 'In Barrier', 'Ex Barrier' and 'Ph\&In Well' are the abbreviation of 'Injection Barrier', 'Extraction Barrier' and 'Phonon \& Injective Well', respectively.

Fig. 2. The calculated GVD. (a) Waveguide loss as a function of frequency and the illustration is a twodimensional model by COMSOL. The inset shows the calculated two-dimensional mode at 4.6THz. (b) Clamped gain and total loss as a function of frequency for devices. (c)Total GVD, Gain GVD, Material GVD and Waveguide GVD as a function of frequency.

Fig. 3. QCL in test after the encapsulation.

Fig. 4. The L-I-V characteristics of the $150-\mu \mathrm{m}$-wide and 3-mm-long device at $10-65 \mathrm{~K}$ temperatures in the $\mathrm{cw}$ mode.

Fig. 5. The $\mathrm{CW}$ emission spectra of the 3-mm-long device at $10 \mathrm{~K}$ at various injection current. The red dashed lines show the noise floor of the spectra.

Fig. 6. (a) Free running beatnote mapping as a function of drive current measured at $10 \mathrm{~K}$ in $\mathrm{CW}$ mode. The resolution bandwidth is set as $300 \mathrm{KHz}$. (b) All beatnote mapping at the current of 0.3-0.7A with an RF spectrum analyzer (resolution bandwidth (RBW): $300 \mathrm{KHz}$, video bandwidth (VBW): $3 \mathrm{KHz}$ ). (c) The narrowest beatnote linewidth at 0.6 Awith an RF spectrum analyzer (RBW: 100KHz, and VBW: $1 \mathrm{KHz}$ ).

Fig. 7. (a)Without temperature control, temperature as a function of current. (b)The simulated gain as a function of temperature. 


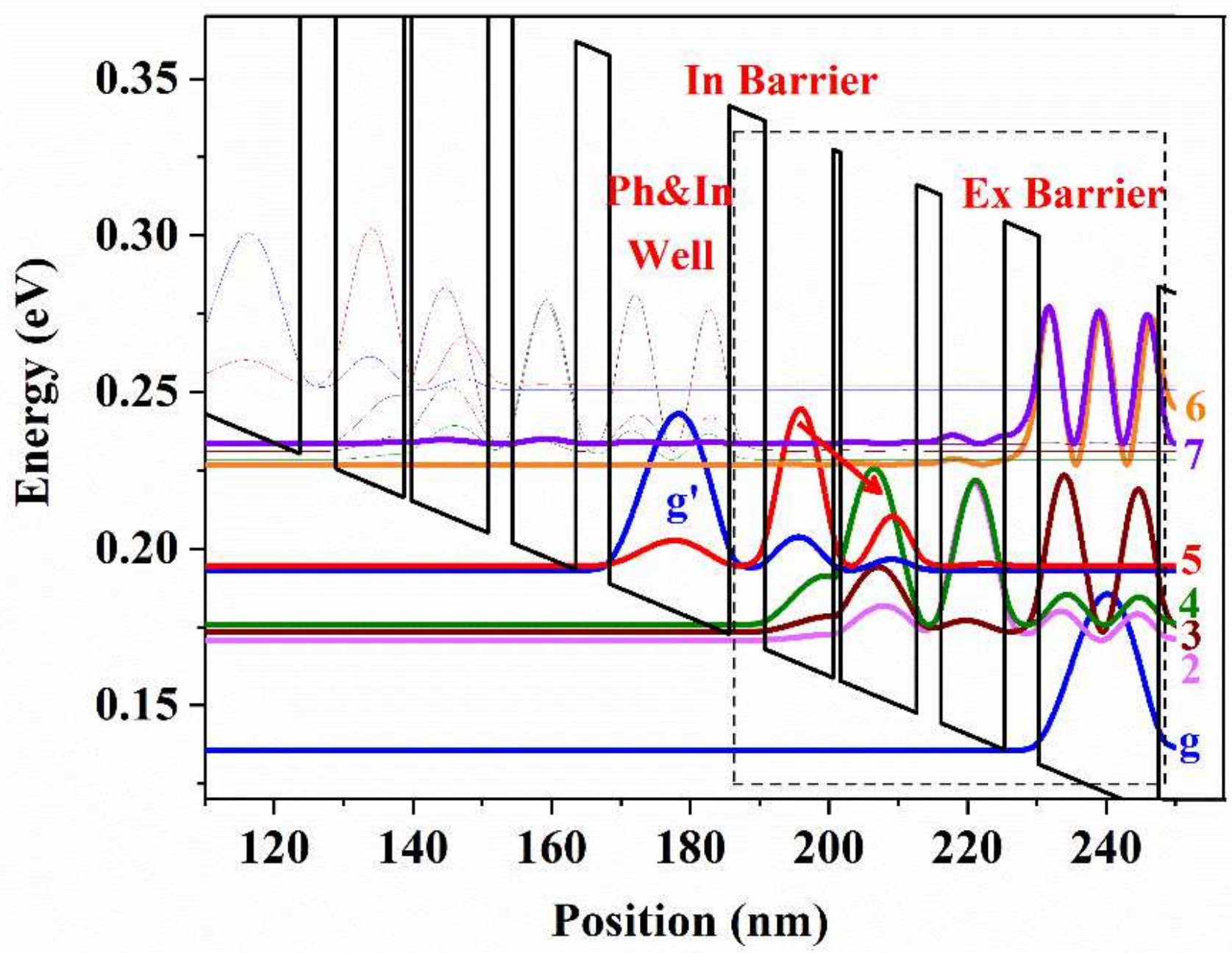

Figure 1

Conduction band diagram and energy level profile of a 4.6 THz QCL structure. An electric field of 9.3 $\mathrm{kV} / \mathrm{cm}$ is applied to the structure. The red arrow marks the radiative transition. The upper laser level (level 5) and the lower lasing level (level 4) are shown in red and green, respectively. The ground state of the injector (level g) is shown in blue. 'In Barrier', 'Ex Barrier' and 'Ph\&In Well' are the abbreviation of 'Injection Barrier', 'Extraction Barrier' and 'Phonon \& Injective Well', respectively. 
(a)

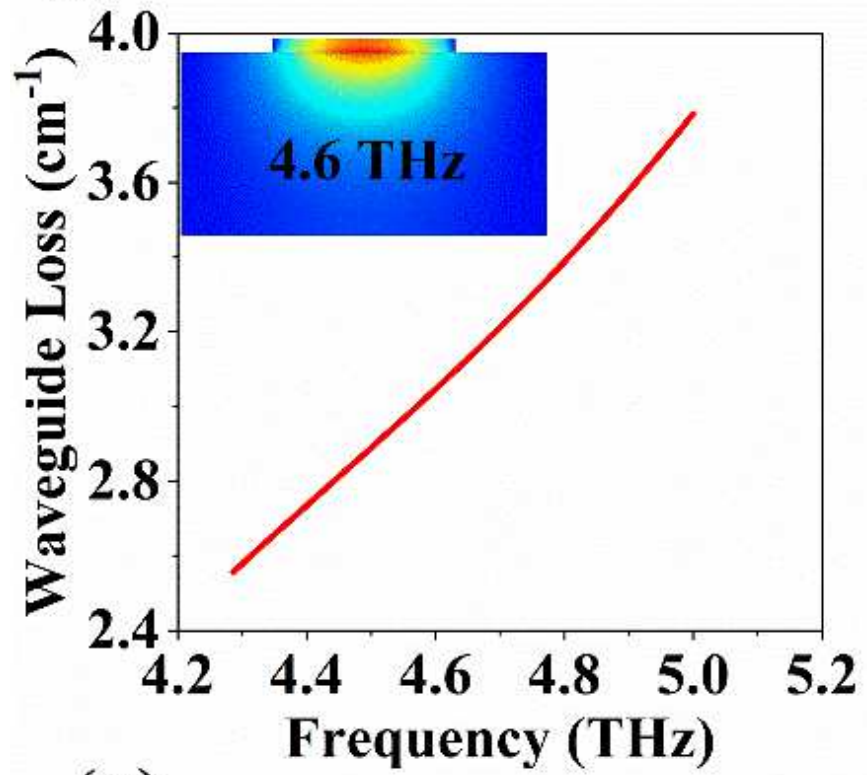

(c)

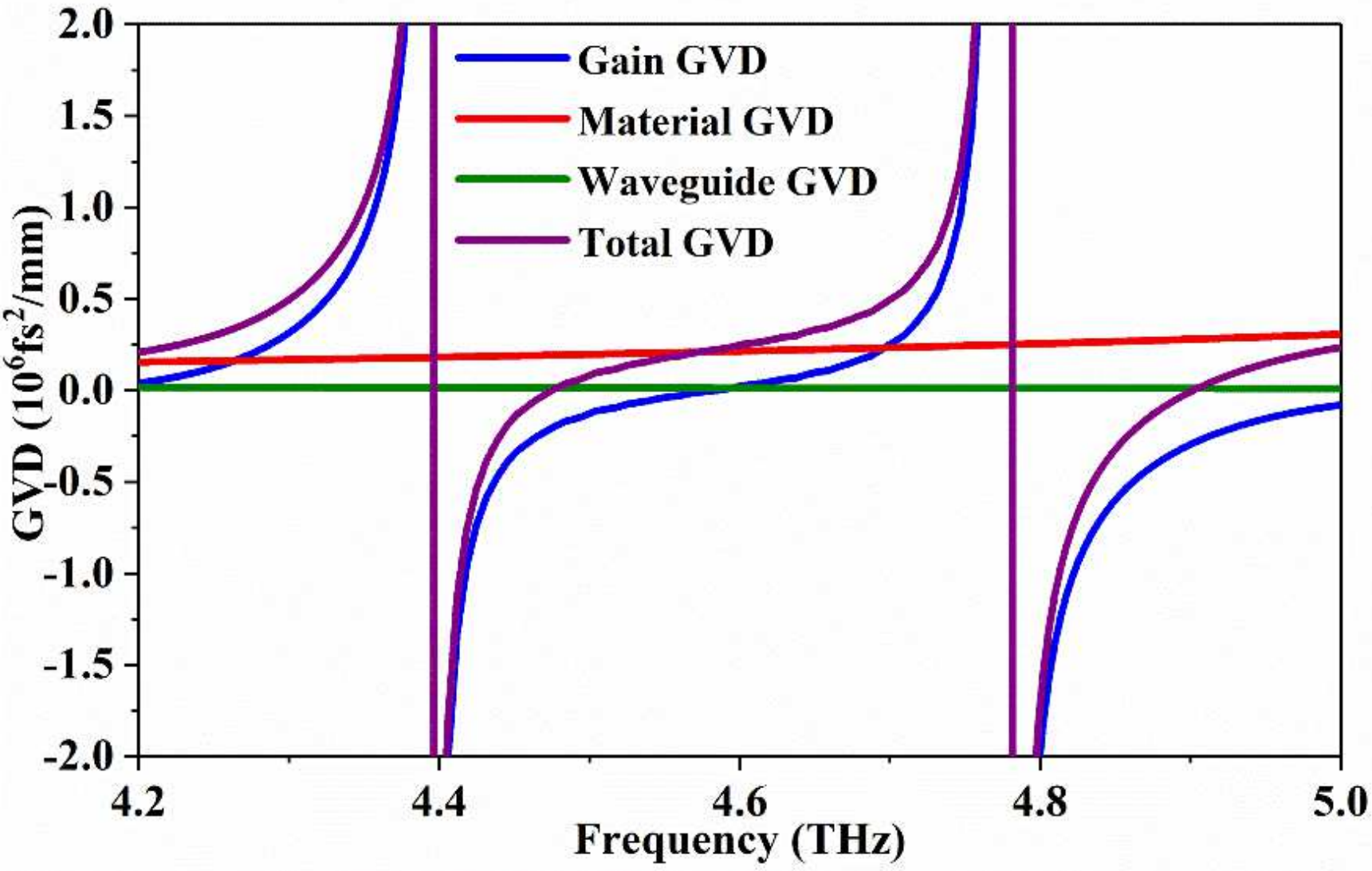

Figure 2

The calculated GVD. (a) Waveguide loss as a function of frequency and the illustration is a twodimensional model by COMSOL. The inset shows the calculated two-dimensional mode at 4.6THz. (b) Clamped gain and total loss as a function of frequency for devices. (c)Total GVD, Gain GVD, Material GVD and Waveguide GVD as a function of frequency. 


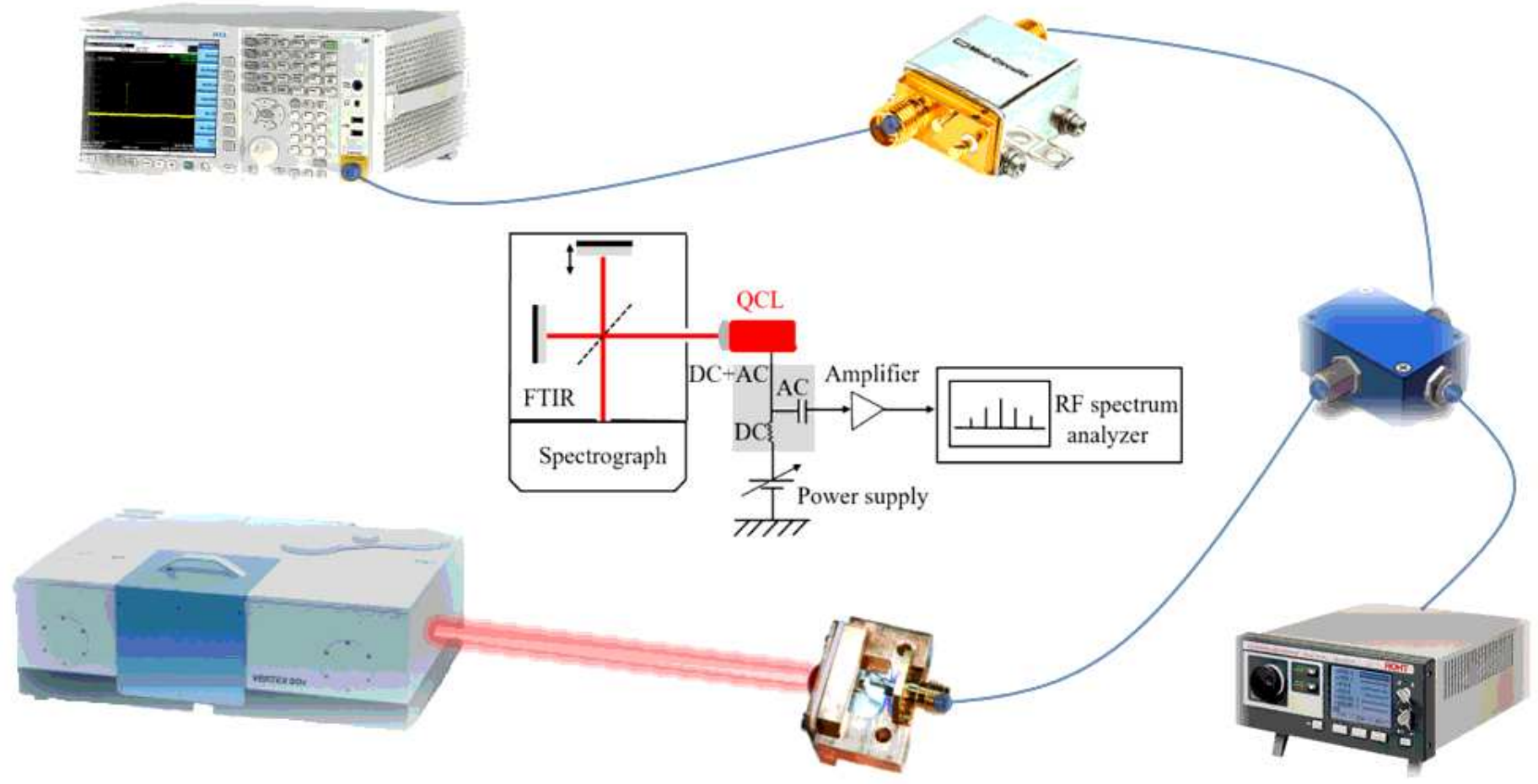

Figure 3

QCL in test after the encapsulation. 


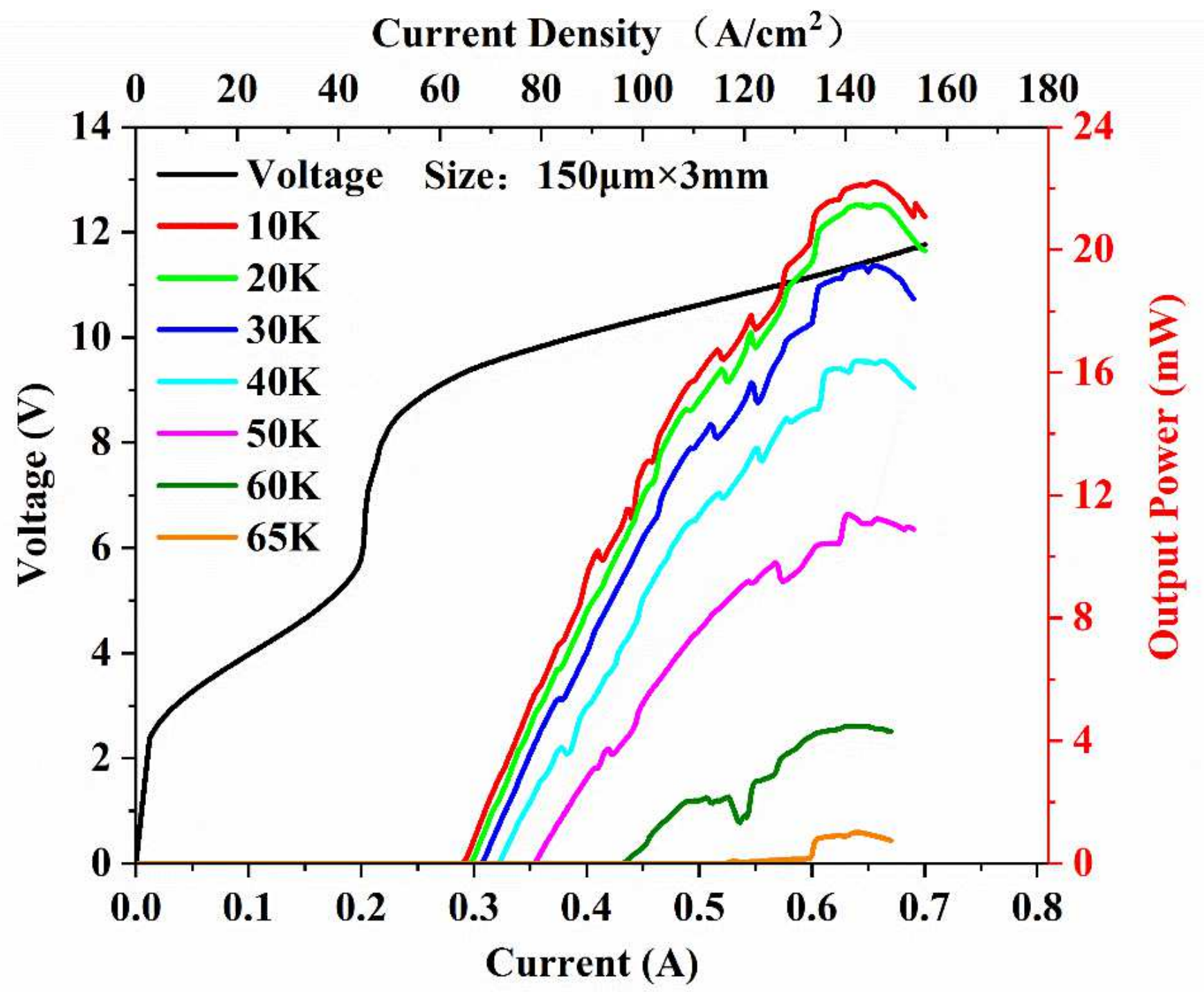

Figure 4

The L-I-V characteristics of the 150- $\mu \mathrm{m}$-wide and 3-mm-long device at $10-65 \mathrm{~K}$ temperatures in the $\mathrm{cW}$ mode. 

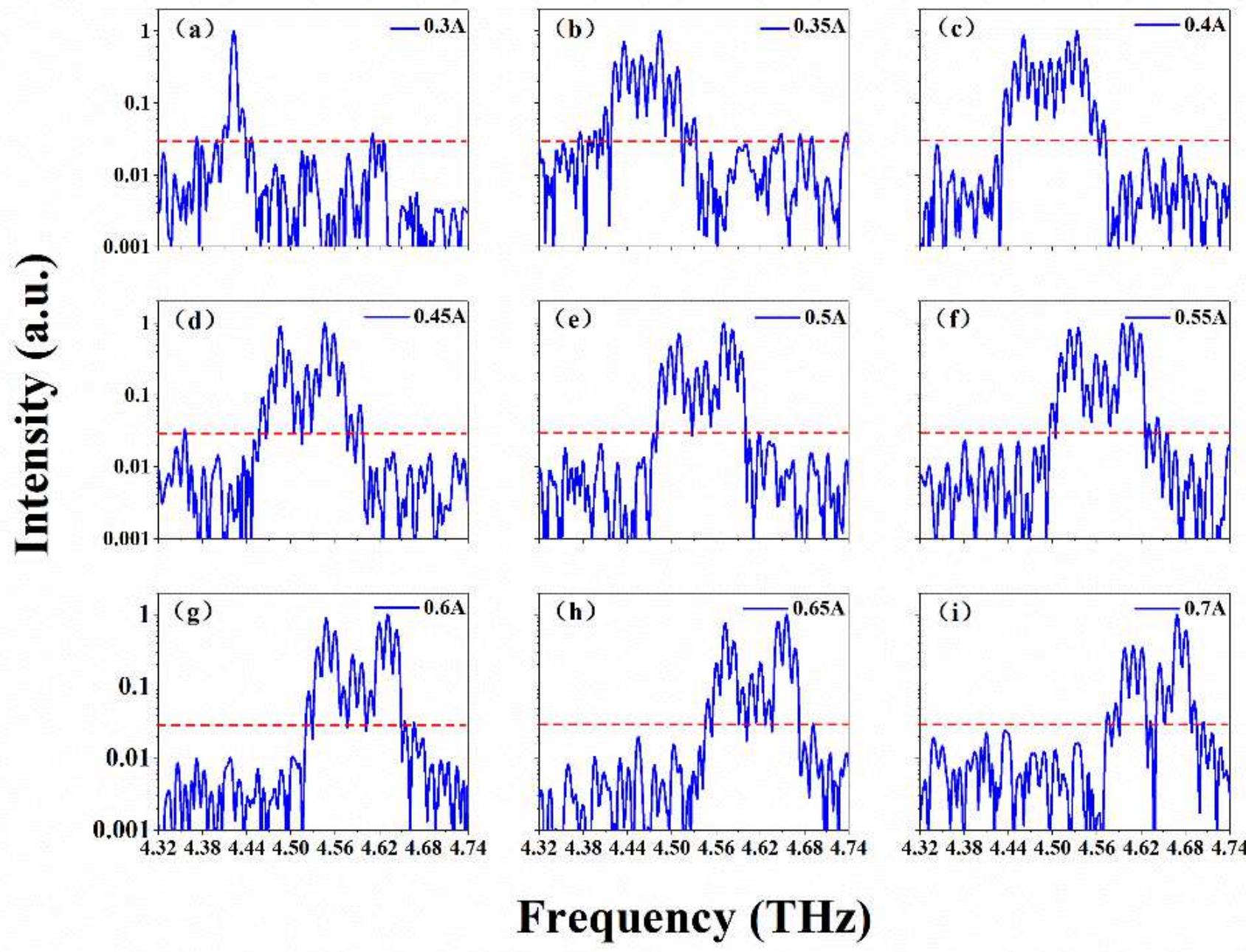

Figure 5

The $\mathrm{CW}$ emission spectra of the 3-mm-long device at $10 \mathrm{~K}$ at various injection current. The red dashed lines show the noise floor of the spectra. 
(a)

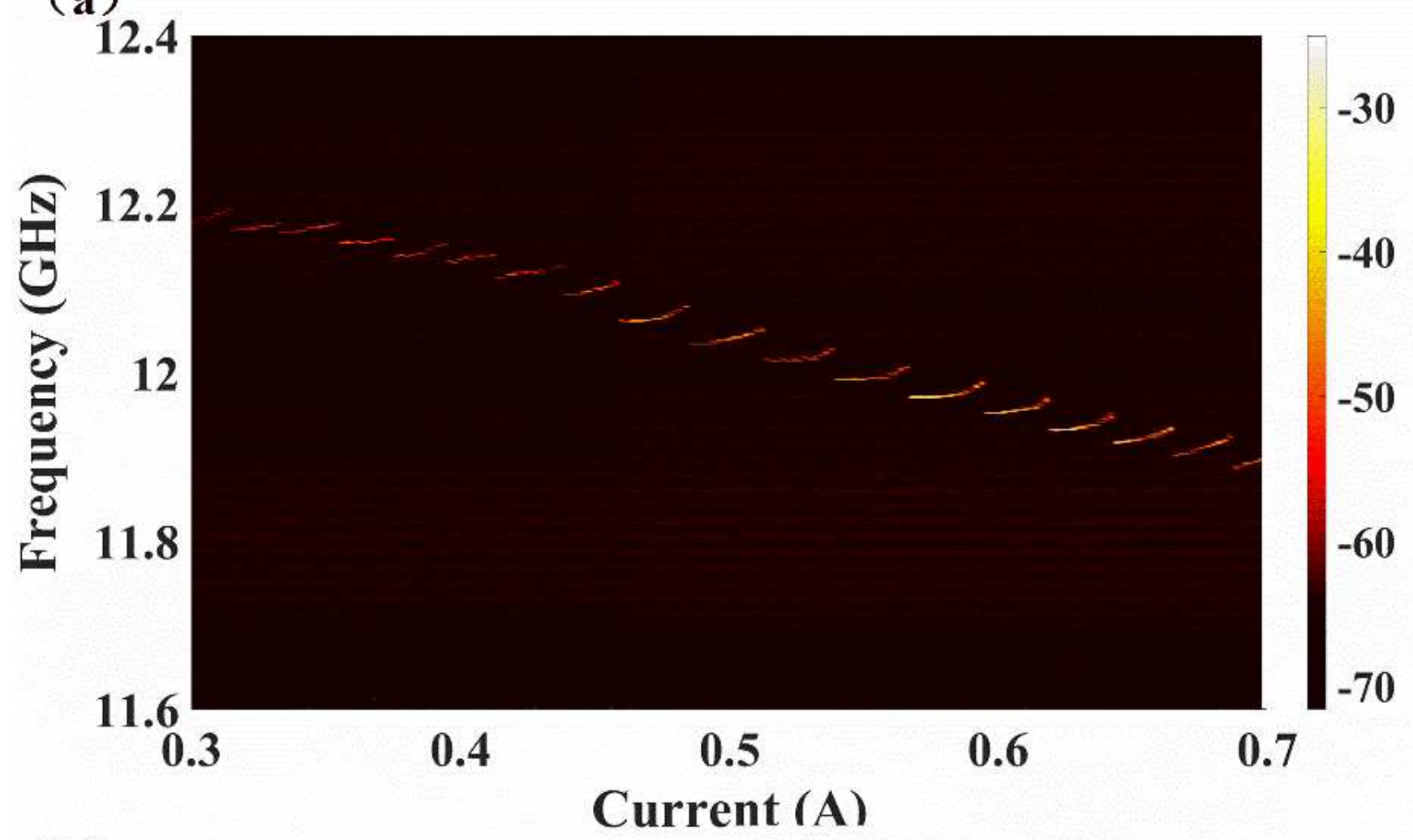

(b)

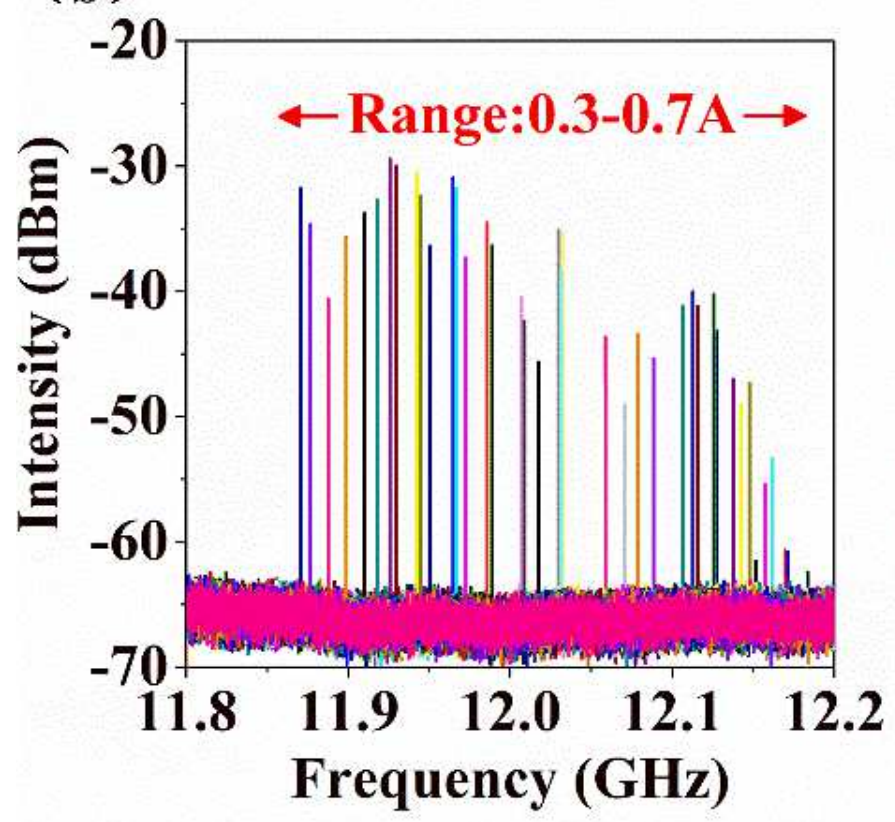

(c)

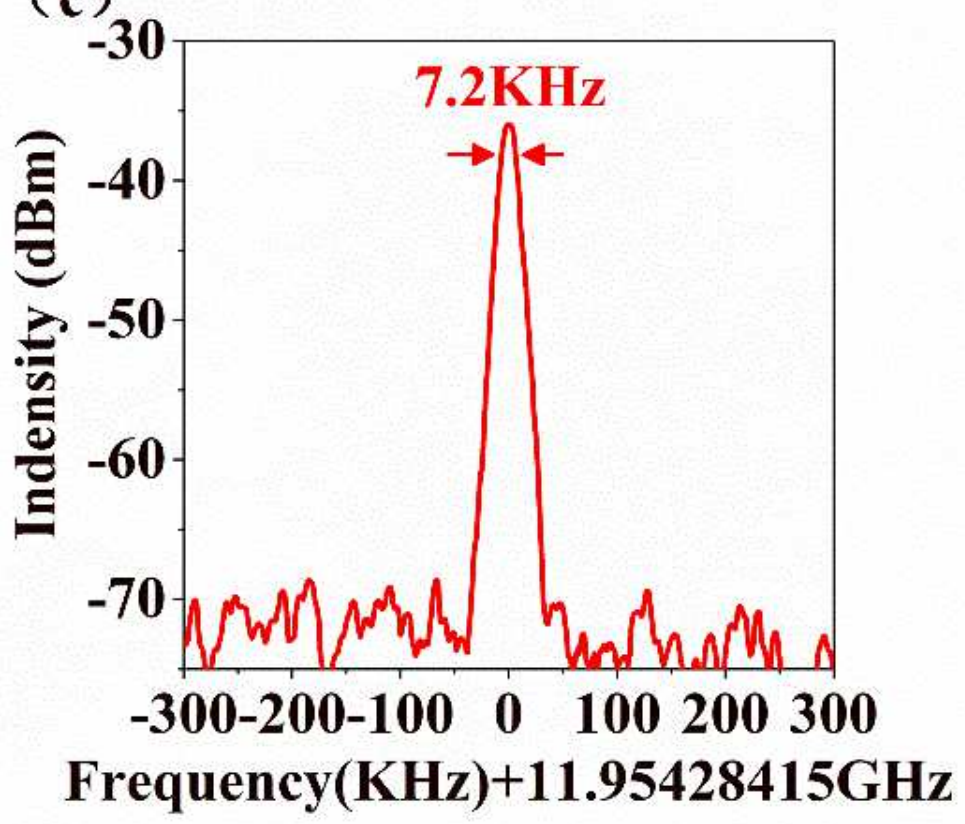

Figure 6

(a) Free running beatnote mapping as a function of drive current measured at $10 \mathrm{~K}$ in $\mathrm{CW}$ mode. The resolution bandwidth is set as $300 \mathrm{KHz}$. (b) All beatnote mapping at the current of 0.3-0.7A with an RF spectrum analyzer (resolution bandwidth (RBW): $300 \mathrm{KHz}$, video bandwidth (VBW): $3 \mathrm{KHz}$ ). (c) The narrowest beatnote linewidth at $0.6 \mathrm{~A}$ with an RF spectrum analyzer (RBW: $100 \mathrm{KHz}$, and VBW: $1 \mathrm{KHz}$ ). 
(a)

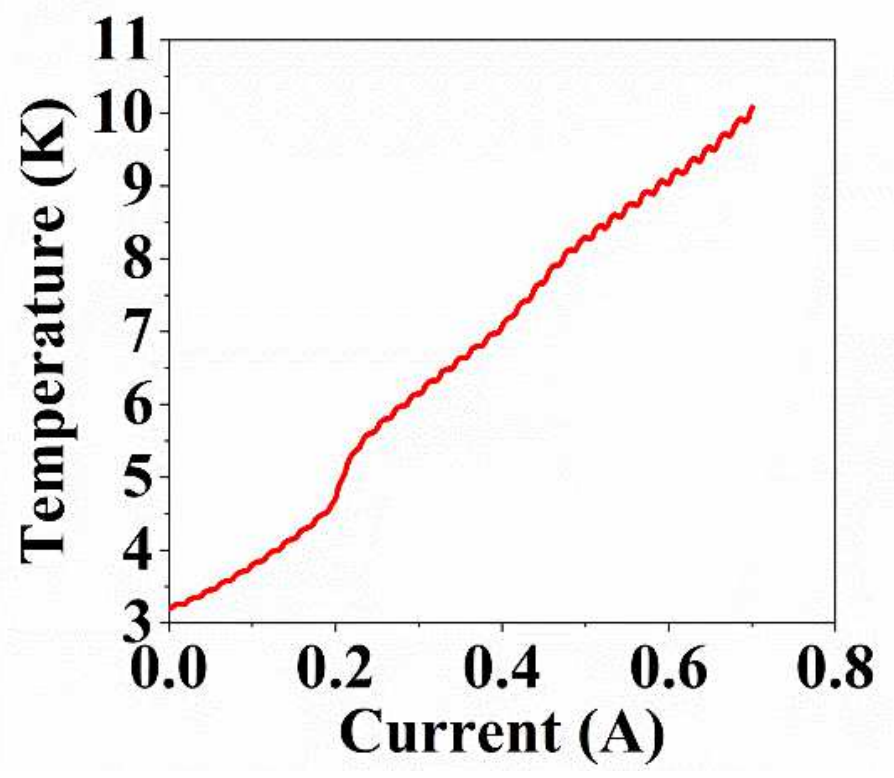

(b)

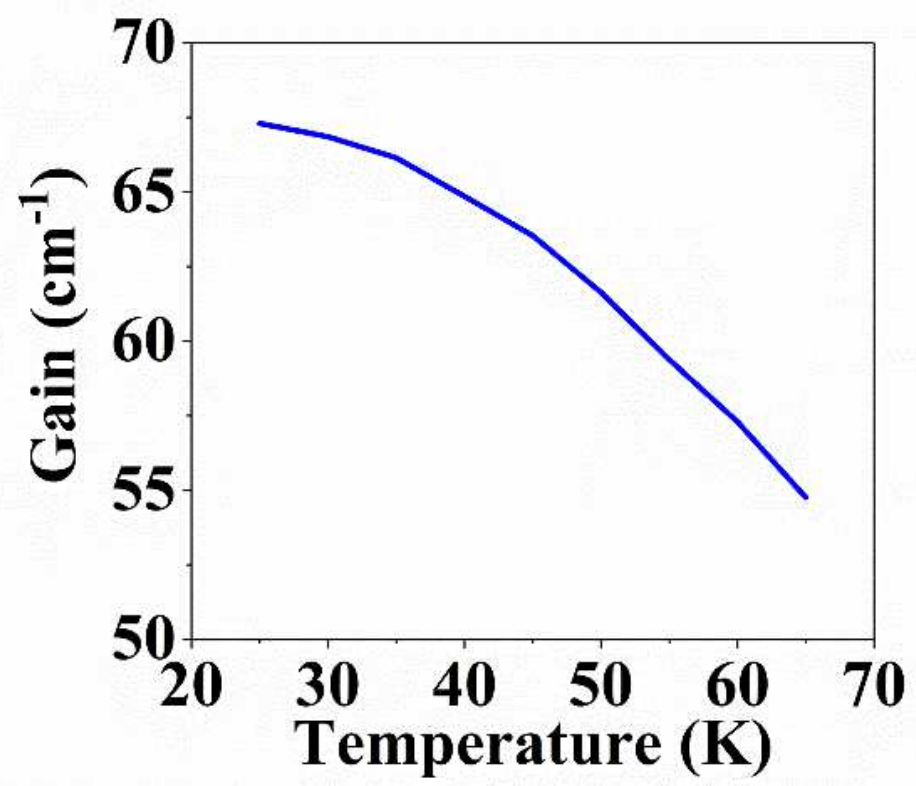

Figure 7

(a)Without temperature control, temperature as a function of current. (b)The simulated gain as a function of temperature. 